Revue belge de géographie

1 | 2008

Aspects of the history of the Belgian geography and cartography

\title{
Laconte P. \& Hein C. (Ed.) Brussels. Perspectives on a European Capital
}

Foundation For the Urban Environment (FFUE), 2007, 127 p.

\section{Patrick Frenay}

\section{(Q) OpenEdition \\ Journals}

Édition électronique

URL : http://journals.openedition.org/belgeo/10215

DOI : $10.4000 /$ belgeo.10215

ISSN : 2294-9135

Éditeur :

National Committee of Geography of Belgium, Société Royale Belge de Géographie

Édition imprimée

Date de publication : 31 mars 2008

Pagination : 137-138

ISSN : 1377-2368

Référence électronique

Patrick Frenay, «Laconte P. \& Hein C. (Ed.) Brussels. Perspectives on a European Capital », Belgeo [En ligne], 1 | 2008, mis en ligne le 19 octobre 2013, consulté le 22 septembre 2020. URL : http:// journals.openedition.org/belgeo/10215; DOI : https://doi.org/10.4000/belgeo.10215

Ce document a été généré automatiquement le 22 septembre 2020.

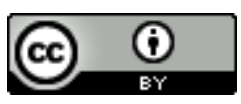

Belgeo est mis à disposition selon les termes de la licence Creative Commons Attribution 4.0 International. 


\title{
Laconte P. \& Hein C. (Ed.) Brussels. Perspectives on a European Capital
}

\author{
Foundation For the Urban Environment (FFUE), 2007, 127 p.
}

\section{Patrick Frenay}

\section{RÉFÉRENCE}

Laconte P. \& HEIN C. (Ed.), Brussels. Perspectives on a European Capital, Foundation For the Urban Environment (FFUE), 2007, 127 p.

1 Cet ouvrage ne pourra être ignoré de l'ensemble du monde des décideurs, planificateurs et "penseurs" en tous genres, actifs en relation avec la Région de Bruxelles-Capitale et la place de cette ville-région dans l'armature urbaine européenne.

2 Il s'ouvre par une large page d'histoire retraçant des étapes fondamentales du développement historique de Bruxelles, pesant bien les éléments majeurs de nature politique, économique, symbolique, sociale voire culturelle qui l'ont appuyé. L'historique de la présence des principales institutions européennes à Bruxelles est tout à fait fondamental parce qu'il identifie bien les aspects majeurs que l'histoire récente explique pour amener ensuite les défis principaux qui se posent aujourd'hui à l'intégration des fonctions européennes (et toutes les activités qui gravitent autour) dans la dynamique de développement de Bruxelles. La vision qui est présentée indique particulièrement bien comment les "petits pas" successifs (parce que non officiels ou non planifiés) ont abouti à des transformations majeures de Bruxelles. Nous aurions néanmoins aimé en savoir encore davantage à propos de l'implication (financière et opérationnelle) des pouvoirs publics belges qui ont contribué directement ou indirectement parfois très fortement à la présence de ces institutions à Bruxelles. Nous aurions aussi trouvé logique de mettre en évidence les impacts des réformes institutionnelles belges qui sont intervenues durant la seconde moitié du $\mathrm{XX}^{\mathrm{e}}$ siècle pour expliquer les processus décisionnels, de planification urbaine, voire opérationnels. 
3 L'aspect "culturel" hérité parfois de l'histoire européenne très ancienne est très intéressant parce qu'il replace de nombreux choix planologiques et urbanistiques actuels en regard de dynamiques et choix passés, dont une remise en perspective avec les enjeux actuels est très éclairante.

4 La contribution de B. Clerbaux est tout à fait majeure dans cet ouvrage. Il apporte à l'évidence un point de vue très ancré dans la réalité locale qu'il maîtrise parfaitement compte tenu de son expérience spécifique, à divers égards. Il pose dès lors très bien les principaux atouts et faiblesses du développement de la Région de Bruxelles-Capitale, à différents points de vue très pertinents, spécialement par rapport à son rôle de capitale européenne dorénavant officielle (depuis 1992, accord sur la localisation des sièges des institutions de l'UE). Sa contribution fourmille ensuite d'idées concernant un développement urbanistique plus "approprié" du quartier européen à Bruxelles, idées parfois extrêmement novatrices voire "utopiques". Leur intérêt est toujours manifeste mais le lecteur qui n'est pas nécessairement lui-même un spécialiste de son niveau peut se laisser excessivement (?) séduire par son enthousiasme et le pragmatisme (très apparent) de très nombreuses propositions qu'il avance. Il a le grand mérite de montrer que de méga-institutions, présentes dans le tissu urbain hérité (au contraire de nombreux autres exemples, comme Luxembourg voire Strasbourg) pourraient être assez bien intégrées au tissu urbain et que leur présence est positive bien plus que l'inverse, même si cette présence pose (encore) de nombreuses difficultés et choix; choix posés qui sont assurément une énorme difficulté à assumer, comme chaque lecteur pourra le deviner.

5 Pour conclure, les deux auteurs principaux de l'ouvrage posent fort bien la question fondamentale du positionnement international de Bruxelles - singulièrement européen bien sûr - par rapport aux différents niveaux institutionnels considérés, singulièrement par rapport au contexte belgo-bruxellois. Entre les lignes, tous ceux qui en sont suffisamment immergés peuvent lire le potentiel formidable que cette villerégion offre, tant aux niveaux belge qu'européen.

6 Nous regrettons toutefois différentes imprécisions (de forme voire de fond) qui caractérisent en particulier un certain nombre de documents graphiques et surtout cartographiques : légendes incomplètes (de sorte que l'on ne peut lire convenablement ces documents alors qu'ils devraient éclairer le lecteur); l'une ou l'autre incohérence dans le mode de représentation cartographique qui fausse parfois excessivement l'analyse et l'interprétation que l'on peut en faire ; vues parfois peu approfondies voire schématiques, qui laissent sur sa fin quant à la faisabilité des idées mises sur la table (ce dernier aspect nous parait d'autant plus dommageable que l'on peut prendre certaines pistes comme "évidentes" alors que seuls ceux qui connaissent un tant soit peu les problématiques liées (et "ignorées" dans l'ouvrage) peuvent porter un regard "juste " sur ces pistes de solutions); nous devons aussi constater quelques erreurs de fond "flagrantes". Nous regretterons aussi l'ancrage déséquilibré du contexte par rapport à différents schémas de développement territorial existants, comme le SDEC, le Schéma Bénélux, le SDER, quasi-absents du présent ouvrage alors qu'ils auraient pu (dû ?) être pris en considération pour que les institutions concernées puissent mieux s'approprier l'ouvrage et surtout les enjeux décisionnels qui se posent en ce début de siècle. 